\title{
LAS ESTRATEGIAS COGNITIVO-CONDUCTUALES Y EXPECTATIVAS SOBRE SUS BENEFICIOS MEDIAN LA RELACIÓN ENTRE APOYO SOCIAL PARENTAL Y FRECUENCIA DE ACTIVIDAD FÍSICA DE LOS ADOLESCENTES
}

\author{
Manuel De la Torre Cruz \\ Departamento de Psicología \\ Universidad de Jaén \\ majecruz@ujaen.es
}

Fecha de Recepción: 7 Febrero 2018

Fecha de Admisión: 10 Abril 2018

\section{RESUMEN}

En los últimos años ha proliferado la idea de concebir la actividad física como una conducta compleja afectada por una multiplicidad de factores. Los enfoques ecológicos son válidos para el análisis de la actividad física infantil y juvenil al permitir incorporar variables de distinta naturaleza (cognitivas, conductuales, interpersonales 0 ambientales). En este estudio, 494 adolescentes (52.2\% chicas), con edades comprendidas entre los 12 y 16 años $\left(M_{\text {edad }}=13.96\right)$ informaron del apoyo social (materno y paterno) para la práctica de actividad física, la frecuencia con la que empleaban estrategias de autocontrol para promover su realización, las expectativas de resultado asociadas a dicha práctica y la cantidad de días a la semana que se implicaban en la realización, durante al menos 60 minutos, de actividad física a una intensidad situada entre moderada y vigorosa. Se examinó si las variables de naturaleza cognitiva (estrategias y expectativas) mediaban en la relación entre el apoyo parental percibido y la frecuencia semanal de práctica de actividad física. Los resultados obtenidos revelaron la existencia de una asociación entre el apoyo social percibido y la frecuencia de práctica de actividad física, relación que estuvo mediada paralelamente por el mayor empleo de estrategias de autocontrol y por unas mayores expectativas de resultado sobre los beneficios vinculados a su práctica.

Palabras clave: apoyo parental; actividad física; estrategias cognitivo-conductuales; expectativas de resultado; adolescentes

\footnotetext{
ABSTRACT:

Cognitive-behavioral strategies, and expectations about their benefits, mediate the relationship between social parental support and physical activity frequency in adolescents.

In recent years the idea of conceiving physical activity as a complex behavior which is influenced
} 
by multiple factors has proliferated. Ecological models are valid frameworks to analyze the physical activity in young, because it allows including different kinds of variables (cognitive, behavioral, interpersonal and environmental variables). In this study, perceived social support to physical activity, the use of self-control strategies and outcome expectancies associated with physical activity and number of days a week, for at least 60 minutes per day, of moderate-to-vigorous physical activity (MVPA) were reported by 494 adolescents (52.2\% girls) aged from 12 to 16 years old. It was examined if the relationship between social parental support and MVPA was mediated by cognitive variables (self-control strategies and outcome expectancies). The obtained results showed a relationship between social parental support and MVPA, relationship which was mediated by a higher use of higher self-control strategies and higher outcomes expectancies about physical activity.

Keywords: parental support; physical activity; cognitive-behavioral strategies; outcome expectancies; adolescents

\section{INTRODUCCIÓN}

La práctica de actividad física ha sido identificada como un elemento trascendente en la mejora de la salud física y psicológica de niños y adolescentes (Baskin, Thind, Affuso, Gary, LaGory, \& Hwang, 2013; Ho, Louie, Chow, Wong, \& Ip, 2015). La realización de actividad física a una intensidad que oscila entre moderada y vigorosa se ha vinculado a una mejor condición física cardio-respiratoria y actividad metabólica, un incremento en la masa ósea, así como niveles más reducidos de sobrepeso y obesidad (Huffman, Wilson, Van Horn, \& Pate, 2018; Powell, Paluch, \& Blair, 2011). Asimismo, la mayor frecuencia de práctica de actividad se ha relacionado con una menor sintomatología depresiva y ansiosa, así como mayor calidad de vida, bienestar subjetivo, capacidad resiliente y fortaleza mental (Moljord, Moksnes, Espnes, Hjemdal, \& Eriksen, 2014; Monshouwer, ten Have, van Poppel, Kemper, \& Vollenbergh, 2013; Sheperd, Krägeloh, Ryan, \& Schofield, 2012).

Realizar actividad física es una acción promovida por una multiplicidad de variables. Además de factores personales como la competencia percibida (Bai, Chen, Vazou, Welk, \& Schaben, 2015; Hands, Parker, Rose, \& Larkin, 2016), el nivel de habilidad motora (Khodaverdi, Bahram, Stodden, \& Kazemnejad, 2015) o la diversión experimentada (Yli-Piipari, Watt, Jaakkola, Liukkonen, \& Nurmi, 2009), los ambientes físicos y sociales actúan como determinantes de interés en la práctica de actividad física de niños y adolescentes (Lawman \& Wilson, 2014). Los modelos ecológicos representan una alternativa teórica útil en la que integrar las relaciones que mantienen entre sí todos estos factores (Silva, Lott, Mota, \& Welk, 2014; Verloigne, Cardon, De Craemer, D'Haese, \& De Bourdeaudhuij, 2016; Welk, 1999). Estos marcos teóricos sugieren que las variables de naturaleza ambiental pueden ejercer una influencia sobre la conducta de niños y adolescentes de forma directa, o bien, de forma indirecta por medio de factores personales (mediadores). Explorar hasta qué punto los factores personales median la relación entre el ambiente y la práctica de actividad física puede ayudar a comprender los procesos que subyacen a dicha práctica y diseñar, en consecuencia, intervenciones más efectivas para incrementarla.

Uno de estos modelos es el de la Promoción de la Actividad Física Juvenil que identifica variables cognitivas (actitudes, creencias, autopercepciones), sociales (apoyo social de padres e iguales) y personales (destreza motora o condición física) entre los correlatos asociados con la actividad física (Bai et al., 2015; Silva et al., 2014; Welk, 1999). Entre las variables de naturaleza social el apoyo parental ha acaparado la atención en un buen número de investigaciones (Brunet et al., 2014; Laird, Fawkner, Kelly, McNamee, \& Niven, 2016; Morrissey, Wenthe, Letuchy, Levy, \& Janz, 2012; Verloigne et al., 2016). El apoyo social parental se define como las acciones tangibles (provisión de transporte, pago de cuotas, compra de equipamiento) e intangibles (mensajes de ánimo, consejos 
ofrecidos, felicitaciones) que madres y padres emprenden para favorecer la implicación y realización de actividad física de sus hijos (Huffman et al., 2018; Mutz \& Albrecht, 2017).

Los estudios previos que han analizado la relación entre apoyo parental y realización de actividad física arrojan resultados contradictorios siendo una de las razones el examen de las relaciones directas entre estas dos variables. Por tanto, las investigaciones recientes examinan el efecto mediador que factores personales como la autoeficacia, diversión, competencia o barreras percibidas pueden desempeñar en la relación entre apoyo parental y práctica de actividad física (Dishman, Dunn, Sallis, Vandenberg, \& Pratt, 2010; Huffman et al., 2018; Verloigne et al., 2016; Wing, Bélanger, \& Brunnet, 2016). Así pues, el objetivo de este estudio consistió en conocer hasta qué punto el empleo de estrategias cognitivas de auto-regulación y las expectativas depositadas en los beneficios de la práctica de actividad física podían mediar en la relación entre apoyo parental percibido y práctica de actividad física en un grupo de adolescentes.

\section{MÉTODO}

\section{Participantes}

Un total de 496 (258 chicas, 52.01\%) estudiantes de Educación Secundaria Obligatoria, con un promedio de edad de 13.97 años (DT = 1.35), participaron en este estudio. Los adolescentes pertenecían a dos centros educativos ubicados en la provincia de Jaén. El peso promedio del conjunto de los participantes fue de 54.21 kilogramos (DT = 11.77), mientras que el de altura se situó en 1.64 metros $(D T=.10)$. A partir de estos valores se calculó el índice de masa corporal tomando como referente la fórmula establecida por Quetelet, $\left(I M C=\right.$ kilogramos/altura en metros $\left.{ }^{2}\right)$. El valor promedio obtenido fue de 19.96 (DT = 3.38).

\section{Instrumentos}

Estrategias de autocontrol cognitivo. Las estrategias de autocontrol fueron evaluadas empleando la escala desarrollada por Dishman et al., (2005). El instrumento consta de ocho ítems, cuatro que reflejan el uso de estrategias cognitivas (p.e.: "Intento pensar más en los beneficios que en las molestias de practicar actividad física") y otros cuatro que valoran el uso de estrategias conductuales ("Hago cosas para que la práctica de actividad física resulte más divertida"). Los participantes han de indicar la frecuencia con la que ponen en práctica tales estrategias a lo largo de una escala tipo Likert de cinco puntos ( 1 = nunca, $5=$ casi siempre). Una mayor puntuación equivale a un mayor uso de estrategias. En este estudio solo se empleó la subescala de estrategias de autocontrol cognitivas. El valor de fiabilidad, obtenido mediante el estadístico alpha de Cronbach para esta muestra de estudio, fue de .79 .

Expectativa de resultado (Dishman et al., 2010). Esta dimensión fue valorada por medio de nueve afirmaciones que expresaban posibles beneficios (físicos, afectivos y sociales) asociados a la práctica de actividad física (p.e.: "La práctica de actividad física me ayudaría a controlar mi peso corporal"). Los participantes debían indicar el grado de acuerdo/desacuerdo con cada una de las afirmaciones a través de una escala tipo Likert con cinco opciones de respuesta ( 1 = muy en desacuerdo; 5 = muy de acuerdo). Una mayor puntuación en la escala se equiparó con unos resultados esperados más valorados. El índice de fiabilidad calculado mediante el estadístico alpha de Cronbach para esta muestra de estudio ascendió a .81.

Actividad física a una intensidad que oscila entre moderada y vigorosa. La frecuencia de práctica semanal de actividad física fue evaluada con la escala Moderate-to-vigorous Physical Activity, "MVPA" (Prochaska, Sallis, \& Long, 2001), compuesta por dos ítems. El participante ha de indicar el número de días durante la semana previa, así como el número de días durante una semana cual- 
quiera en la que el participante realizó actividad física a una intensidad que oscila entre moderada y vigorosa durante al menos 60 minutos diarios. La prueba define actividad física en términos genéricos, a saber, como cualquier actividad que incrementa la tasa cardíaca y dificulta la respiración. Se obtiene un valor que es resultado de promediar las respuestas a ambos ítems. Un valor de cinco 0 superior se corresponde con la inclusión del joven en la categoría "físicamente activo". El índice de fiabilidad de los dos ítems en la muestra de estudio ascendió a .81.

Apoyo social parental. El apoyo social, tanto materno como paterno, fue evaluado a partir de una escala compuesta por 10 ítems (Liang, Lau, Huang, Maddison, \& Baranowski, 2014) que se adaptaron a partir de los incluidos en la Escala de Apoyo Social para el Ejercicio (Sallis, Grossman, Pinski, Patterson, \& Nader, 1987). Respecto al instrumento original, Liang et al. (2014), sustituyeron el término "ejercicio físico" por el de "actividad física". Los participantes indicaban el grado de acuerdo mostrado con una serie de afirmaciones relativas al apoyo social que creían haber recibido por parte de madres y padres para la práctica de actividad física a lo largo del último año (p.e.: "Durante el último año, mi madre/padre modificó su horario para que practicásemos actividad física juntos") en una escala tipo Likert de cuatro puntos ( 1 = totalmente en desacuerdo, 4 = totalmente de acuerdo). Una mayor puntuación alcanzada en la escala se correspondía con un nivel más elevado de apoyo social percibido. El índice de fiabilidad calculado mediante el estadístico alpha de Cronbach ascendió a .86 y .91, para madres y padres, respectivamente.

\section{Procedimiento}

Para acceder a la muestra de interés se redactó una carta dirigida a los equipos directivos de dos centros educativos de la provincia de Jaén solicitando su colaboración para la realización de este estudio. En la carta se explicitaba que el objetivo principal residía en explorar las posibles relaciones existentes entre el apoyo que chicos y chicas afirmaban recibir por parte de sus progenitores para la práctica de actividad físico-deportiva y diversas variables relacionadas con dicha participación (frecuencia, disfrute, autoeficacia percibida para la práctica o capacidad para eliminar barreras u obstáculos que dificultan su realización).

Padres o tutores legales de los menores recibían esta información en forma de consentimiento informado que debían remitir firmado autorizando la participación del menor en el estudio. Los diferentes cuestionarios se aplicaron durante alguna de las horas lectivas destinadas a la asignatura de Educación Física. Los participantes dispusieron de 50 minutos para completar las diferentes medidas. El protocolo contó con la aprobación del Comité de Bioética de la Universidad de Jaén.

\section{Análisis estadístico}

En primer lugar, se realizó un análisis descriptivo (media y desviación típica) de las variables incluidas en el estudio. La fiabilidad de las medidas de autoinforme se obtuvo mediante el estadístico alpha de Cronbach. Asimismo, se empleó el índice de correlación de Pearson para examinar la relación entre variables.

El análisis de mediación fue conducido mediante la macro PROCESS (Preacher \& Hayes, 2004), integrada en el programa estadístico SPSS. Concretamente, se pusieron a prueba dos modelos con dos mediadores en paralelo que admiten la posible relación sin que necesariamente tenga que existir una relación causal entre ellos (Hayes, 2013). En ambos modelos la variable independiente fue el apoyo parental percibido (madres y padres, respectivamente) y la variable dependiente la frecuencia semanal de práctica de actividad física. Las variables mediadoras fueron las estrategias de autocontrol y las expectativas de resultados asociadas a la realización de actividad física. El programa estadístico utilizado fue SPSS versión 21.0 para Windows. El nivel de significación estadística se situó en un $p$ valor inferior a 05 . 


\section{RESULTADOS}

En la tabla número 1 se muestran los valores promedio, desviaciones típicas e índices de correlación entre las diferentes medidas empleadas. Los resultados arrojaron la existencia de correlaciones positivas y estadísticamente significativas entre todas y cada una de las variables consideradas. Un mayor apoyo social percibido en madres y padres se asoció a una mayor frecuencia de actividad física semanal ( $r=.19, p<.01$ y $r=.23, p<.01$, respectivamente). De la misma forma el empleo de estrategias cognitivas de autocontrol, así como unas expectativas de resultado más elevadas, mantuvieron una relación positiva y estadísticamente significativa con la frecuencia de actividad física semanal ( $r=.38, p<.01$ y $r=.34, p<.01$, respectivamente).

El análisis de mediación realizado (dos mediadores en paralelo), empleando el método de mínimo cuadrados ordinarios, reveló que el apoyo parental percibido ejercía una influencia indirecta sobre la frecuencia de práctica de actividad física semanal por medio de su efecto sobre las estrategias de auto-regulación cognitiva y las expectativas de resultado de los participantes. Tal y como se observa en la tabla número 2, los jóvenes que atribuyeron un mayor apoyo social a sus madres recurrían con más frecuencia a las estrategias de auto-regulación cognitiva $\left(a_{1}=.354\right)$ y depositaban unas mayores expectativas de resultado asociadas a la práctica de actividad física $\left(a_{2}=.200\right)$ con relación a quienes percibían en sus madres un menor apoyo social. Adicionalmente, los jóvenes que empleaban estrategias de auto-regulación cognitiva con más frecuencia $\left(b_{1}=.489\right)$ y depositaban mayores expectativas de resultado $\left(b_{2}=474\right)$ realizaban actividad física semanal con más frecuencia. El intervalo de confianza con remuestreo corregido de sesgo para los efectos indirectos de las estrategias de autorregulación cognitiva $\left(a_{1} b_{1}=.173[.104-.263]\right)$ y las expectativas de resultado $\left(a_{2} b_{2}=.095[.048-.158]\right)$, basado en 5000 muestras, fue superior al valor de cero en ambos casos. No se evidenció que el apoyo materno afectase a la práctica de actividad física independientemente de su efecto sobre la autorregulación cognitiva y expectativas de resultado $(c=$ .183, $p=.066)$.

Cuando el análisis se centró en la figura del padre (ver tabla número 3), los resultados fueron prácticamente similares. El mayor apoyo social percibido en los padres se vinculó con un uso más elevado de estrategias de auto-regulación cognitiva $\left(a_{1}=.293\right)$ y mayores expectativas de resultado $\left(a_{2}=.155\right)$, estrategias de auto-regulación y expectativas que a su vez influían $\left(b_{1}=.473\right.$ y $b_{2}=$ .467), respectivamente, en la frecuencia semanal de práctica de actividad física. El intervalo de confianza con remuestreo corregido de sesgo para los efectos indirectos de las estrategias de autorregulación cognitiva $\left(a_{1} b_{1}=.138[.084-.215]\right)$ y las expectativas de resultado $\left(a_{2} b_{2}=.072[.035-\right.$ .123]), basado en 5000 muestras, fue superior al valor de cero en ambos casos. Adicionalmente el apoyo paterno influyó en la práctica de actividad física con independencia de los efectos mediadores de la auto-regulación cognitiva y las expectativas de resultado $(c=.245, p=.004)$.

\section{CONCLUSIONES Y DISCUSIÓN}

El objetivo del presente estudio consistió en examinar el posible papel mediador de dos variables personales de naturaleza cognitiva (estrategias de autocontrol y expectativas de resultado) en la relación mantenida entre apoyo social parental y frecuencia de actividad física semanal en una muestra de adolescentes. Los resultados obtenidos revelaron que la relación entre el apoyo parental (materno y paterno) y la práctica de actividad física se encontraba mediatizada por las variables personales descritas. Estos resultados coinciden con el de otras investigaciones que destacan la existencia de variables que median esta relación.

En este sentido, Wing et al. (2016) concluyeron que los padres pueden incidir de forma indirecta sobre la realización de la actividad física de los hijos al influir el apoyo parental a la autoeficacia per- 
cibida y la diversión asociada a la práctica de actividad física dentro y fuera del contexto escolar. Asimismo, Dishman et al. (2010), concluyeron que el apoyo parental percibido mantuvo una relación indirecta con la práctica de actividad física de un grupo de chicas adolescentes, relación que estuvo mediatizada por su asociación inversa con los obstáculos percibidos para la práctica. Concretamente, las chicas que atribuyeron un menor apoyo social percibían mayores barreras para la práctica de actividad física que las chicas que afirmaban sentirse más apoyadas, pero, solo si se atribuían a sí un bajo nivel de autoeficacia para superar tales obstáculos.

En una línea similar, Verloigne et al. (2016) obtuvieron que diversos factores parentales (modelado, co-participación e incitación a la práctica) se asociaron de forma positiva con el nivel de actividad física de un grupo de chicas adolescentes. Por añadido, esta relación positiva estuvo mediatizada por variables personales tales como la autoeficacia atribuida para ser físicamente activa y la percepción de los beneficios vinculados a la diversión, estar rodeadas de amigos o entablar nuevas relaciones como resultado de la práctica.

Nuestros hallazgos están en consonancia con lo predicho desde el enfoque socio-ecológico de la actividad física, el cual establece que los factores ambientales pueden ejercer una influencia directa sobre la actividad física, así como otra de carácter indirecto por medio de variables personales. Desde un punto de vista eminentemente práctico destinado a mejorar las intervenciones que promueven un cambio conductual hacia el incremento de la actividad física de niños y adolescentes, parece necesario promover estrategias que puedan ser empleadas por los padres para favorecer la regulación cognitiva junto a una percepción más positiva de los beneficios vinculados al hecho de ser un individuo físicamente activo (Wing et al., 2016).

Finalmente, el estudio presenta algunas limitaciones que han de ser tenidas en cuenta y que requiere que los resultados sean interpretados con cautela. En primer lugar, la naturaleza transversal del diseño empleado impide establecer conclusiones sobre causalidad o direccionalidad. En segundo lugar, los adolescentes informaban del apoyo recibido y número de días a la semana de práctica de actividad física. Como con cualquier medida de autoinforme existe un riesgo de deseabilidad social o sesgo en el recuerdo que puede infra o sobreestimar el valor de la información aportada.

\section{REFERENCIAS BIBLIOGRÁFICAS}

Bai, Y., Chen, S., Vazou, S., Welk, G. J., \& Schaben, J. (2015). Mediated effects of perceived competence on youth physical activity and sedentary behavior. Research Quarterly for Exercise and Sport, 86, 406-413. doi:10.1080/02701367.2015.1087639

Baskin, M. L., Thind, H., Affuso, O., Gary, L. C., LaGory, M., \& Hwang, S. (2013). Predictors of moderate-to-vigorous physical activity (MVPA) in African American young adolescents. Annual Behavioral Medicine, 45, S142-S150.doi:10.1007/s12160-012-9437

Brunet, J., Sabiston, C. M., 0 Loughlin, J., Mathieu, M. E., Tremblay, A., Barnett, T. A. \& Lambert, M. (2014). Perceived parental social support and moderate-to-vigorous physical activity in children at risk of obesity. Research Quarterly of Exercise and Sport, 85(2), 198-207. Doi:10.1080/02701367.2014.893049.

Dishman, R. K., Dunn, A. L., Sallis, J. F., Vandenberg, R. J., \& Pratt, C. A. (2010). Social-cognitive correlates of physical activity in a multi-ethnic cohort of middle-school girls: Two-year prospective study. Journal of Pediatric Psychology, 35(2), 188-198.

Dishman, R. K., Hales, D. P., Sallis, J. F., Saunders, R., Dunn, A. L., Bedimo-Rung, A. L., \& Ring, K. B. (2010). Validity of social-cognitive measures for physical activity in middle-school girls. Journal of Pediatric Psychology, 35(1), 72-88. doi:10.1093/jpepsy/jsp031 
Dishman, R. K., Motl, R. W., Sallis, J. F., Dunn, A. L., Birnbaum, A. S., Welk, G. J., et al. (2005). Selfmanagement strategies mediate self-efficacy and physical activity. American Journal of Preventive Medicine, 29, 10-18.

Hands, B., Parker, H. E., Rose, E., \& Larkin, D. (2016). Gender and motor competence affects perceived likelihood and importance of physical activity outcomes among 14 year olds. Child: Care, Health and Development, 42(2), 246-252.

Hayes, A. F. (2013). Introduction to mediation, moderation, and conditional process analysis. A regression-based approach. New Hork, NY. The Guilford Press.

Ho, F. K. W., Louie, L. H. T., Chow, C. B., Wong, W. H. S., \& Ip, P. (2015). Physical activity improves mental health through resilience in Hong Kong Chinese adolescents. BMC Pediatrics, 15:48. doi: 10.1186/212887-015-0365-0

Huffman, L. E., Wilson, D. K., Van Horn, M. L., \& Pate, R. R. (2018). Associations between parenting factors, motivation, and physical activity in overweight African American adolescents. Annals of Behavior Medicine, 52(2), 93-105.

Khodaverdi, Z., Bahram, A., Stodden, D., \& Kazemnejad, (2016). The relationship between actual motor competence and physical activity in children: Mediating roles of perceived motor competence and health-related physical fitness. Journal of Sports Science, 34(16), 1523-1529.

Laird, Y., Fawkner, S., Kelly, P., McNamee, L., \& Niven, A. (2016). The role of social support on physical activity behavior in adolescents' girls: a systematic review and meta-analysis. International Journal of Behavioral Nutrition and Physical Activity, 7,13:19. doi:10.1186/s12996-016-0405-7

Lawman, H. G., \& Wilson, D. K. (2014). Associations of social and environmental supports with sedentary behavior, light and moderate-to-vigorous physical activity in obese underserved adolescents. International Journal of Behavioral Nutrition and Physical Activity, 11:92

Liang, Y., Lau, P. W. C., Huang, W. Y. J., Maddison, R. \& Baranowski, T. (2014). Validity and reliability of questionnaires measuring physical activity self-efficacy, enjoyment, social support among Hong Kong Chinese children. Preventive Medicine Reports, 1, 48-52.

Moljord, I. E. O., Moksnes, U. K., Espnes, G. A., Hjemdal, 0., \& Eriksen, L. (2014). Physical activity, resilience, and depressive symptoms in adolescence.Mental Health and Physical Activity, 7, 7985. doi:10.1016/j.mhpa.2014.04.001

Monshouwer, K., ten Have, M., van Poppel, M., Kemper, H., \& Vollebergh, W. (2013). Possible mechanisms explaining the association between physical activity and mental health: Finding from the 2001 Dutch health behavior in school-aged children survey. Clinical Psychological Science, 1, 67-74.

Morrissey, J. L., Wenthe, P. J., Letuchy, E. M., Levy, S. M., \& Janz, K. F. (2012). Specific types of family support and adolescent non-school physical activity levels. Pediatric and Exercise Science, 24, 333-346.

Mutz, M., \& Albrecht, P. (2017). Parents social status and children s daily physical activity: The role of familial socialization and support. Journal of Child and Family Studies, 26(11), 3026-3035.

Powell, K. E., Paluch, A.E., \& Blair, S. N. (2011). Physical activity for health: What kind? How much? How intense? On top of what? Public Health, 32, 49-365.

Preacher, K. J., \& Hayes, A. F. (2004). SPSS and SAS procedures for estimating indirect effectis in simple mediation models. Behavior Research Methods, Instruments, and Computers, 36, 717731.

Prochaska, J. J., Sallis, J. F., \& Long, B. (2001). A physical activity screening measure for use with adolescents and primary care. Archives of Pediatrics and Adolescents Medicine, 155, 554-559.

Sallis, J. F., Grossman, R. M., Pinski, R. B., Patterson, T. L., \& Nader, P. R. (1987). The development 
of scales to measure social support for diet and exercise behaviors. Preventive Medicine, 16, 825-836.

Shepherd, D., Krägeloh, C., Ryan, C., \& Schofield, G. (2012). Psychological well-being, self-reported physical activity levels, and attitudes to physical activity in a sample of New Zealand adolescent females. Psychology, 3, 447-453.

Silva, P., Lott, R., Mota, J., \& Welk, G. (2014). Direct and indirect effects of social support on youth physical activity behaviour. Pediatric Exercise Science, 26, 86-94. doi:10.1123/pes.2012-0207

Verloigne, M., Cardon, G., De Craemer, M., D’Haese, S., \& De Bourdeaudhuij, I. (2016). Mediating effects of self-efficacy, benefits and barriers on the association between peer and parental factors and physical activity among adolescent girls with a lower educational level. PLOS ONE, 11:6, e0157216

Welk, G. (1999). The youth physical activity promotion model: A conceptual bridge between theory and practice. Quest, 51, 5-23.

Wing, E. K., Bélanger, M., \& Brunet, J. (2016). Linking parental influences and youth participation in physical activity in- and out-of-school: The mediating role of self-efficacy and enjoyment. American Journal of Health Behavior, 40(1), 31-37.

Yli-Piipari, S., Watt, A., Jaakkola, T., Liukkonen, J., \& Nurmi, J. (2009). Relationships between physical education students motivational profiles, enjoyment, state anxiety, and self-reported physical activity. Journal of Sports Science and Medicine, 8, 327-336.

Tabla 1: Medias, desviaciones típicas y valores del índice de correlación de Pearson entre las diferentes variables consideradas en el estudio.

\begin{tabular}{lccccccc}
\cline { 2 - 8 } & Media & DT & 1 & 2 & 3 & 4 & 5 \\
\hline Apoyo social madre & 2.20 & .69 & 1 & & & & \\
Apoyo social padre & 2.24 & .80 & $.67 * *$ & 1 & & & \\
Estrategias cognitivas & 3.96 & .87 & $.39 * *$ & $.39 * *$ & 1 & & \\
Expectativas de resultado & 4.25 & .63 & $.28 * *$ & $.27 * *$ & $.50^{* *}$ & 1 & \\
Actividad física semanal & 3.21 & .59 & $.19 * *$ & $.23 * *$ & $.38^{* *}$ & $.34 * *$ & 1 \\
\hline$* p<.05, * * p<.01$ & & & & & & &
\end{tabular}

Tabla 2: Valor de los coeficientes y significación estadística del análisis de regresión entre el apoyo social materno y la práctica de actividad física semanal empleando como variables mediadoras el empleo de estrategias cognitivas y las expectativas de resultado asociadas a la práctica de actividad física.

\begin{tabular}{|c|c|c|c|c|c|c|c|c|c|c|c|c|}
\hline & \multicolumn{4}{|c|}{ M1 (Estrategias cognitivas) } & \multicolumn{4}{|c|}{ M2 (Expectativas de resultado) } & \multicolumn{4}{|c|}{ Y (Actividad fisica semanal) } \\
\hline Antecedente & & Coeff. & SE & $p$ & & Coeff. & SE & $p$ & & Coeff. & SE & $p$ \\
\hline X (Apoyo social materno) & $a_{1}$ & .354 & .126 & $<.001$ & $a_{2}$ & .200 & .041 & $<.001$ & $c^{\prime}$ & .183 & .099 & .066 \\
\hline $\mathrm{M}_{\mathrm{l}}$ (Estrategias cognitivas) & & & & & & & & & $b_{1}$ & .489 & .089 & $<.001$ \\
\hline $\mathrm{M}_{2}$ (Expectativas de resultado) & & & & & & & & & $b_{2}$ & .474 & .119 & $<.001$ \\
\hline \multirow[t]{2}{*}{ Constante } & $i_{\mathrm{Ml}}$ & 3.187 & .126 & $<.001$ & $i_{\mathrm{M} 2}$ & 3.804 & .094 & $<.001$ & $i_{\mathrm{Y}}$ & -1.141 & .462 & $=.014$ \\
\hline & \multicolumn{4}{|c|}{$\mathrm{R}^{2}=.079$} & \multicolumn{4}{|c|}{$\mathrm{R}^{2}=.047$} & \multicolumn{4}{|c|}{$\mathrm{R}^{2}=.180$} \\
\hline
\end{tabular}




\section{EL PAPEL DE LA PSICOLOGÍA HOY}

Tabla 3: Valor de los coeficientes y significación estadística del análisis de regresión entre el apoyo social materno y la práctica de actividad física semanal empleando como variables mediadoras el empleo de estrategias cognitivas y las expectativas de resultado asociadas a la práctica de actividad física.

\begin{tabular}{|c|c|c|c|c|c|c|c|c|c|c|c|c|}
\hline & \multicolumn{4}{|c|}{ M1 (Estrategias cognitivas) } & \multicolumn{4}{|c|}{ M2 (Expectativas de resultado) } & \multicolumn{4}{|c|}{ Y (Actividad fisica semanal) } \\
\hline Antecedente & & Coeff. & SE & $p$ & & Coeff. & $\mathrm{SE}$ & $p$ & & Coeff. & SE & $p$ \\
\hline X (Apoyo social paterno) & $a_{1}$ & .293 & .047 & $<.001$ & $a_{2}$ & .155 & .084 & $<.001$ & $c^{\prime}$ & .245 & .085 & $=.004$ \\
\hline $\mathrm{M}_{1}$ (Estrategias cognitivas) & & & & & & & & & $b_{1}$ & .473 & .088 & $<.001$ \\
\hline $\mathrm{M}_{2}$ (Expectativas de resultado) & & & & & & & & & $b_{2}$ & .467 & .118 & $<.001$ \\
\hline \multirow[t]{2}{*}{ Constante } & $i_{\mathrm{MI}}$ & 3.308 & .047 & $<.001$ & $i_{\mathrm{M} 2}$ & 3.898 & .084 & $<.001$ & $i_{\mathrm{Y}}$ & -1.192 & .456 & $=.009$ \\
\hline & \multicolumn{4}{|c|}{$\mathrm{R}^{2}=.072$} & \multicolumn{4}{|c|}{$\mathrm{R}^{2}=.038$} & \multicolumn{4}{|c|}{$\mathrm{R}^{2}=.188$} \\
\hline
\end{tabular}


